\title{
Correction to: Breast cancer patients' satisfaction with individual therapy goals and treatment in a standardized integrative medicine consultancy service
}

Carolin C. Hack ${ }^{1}(1)$. Sophia Antoniadis ${ }^{1}$ - Janina Hackl ${ }^{1}$. Hanna Langemann ${ }^{1}$ - Judith Schwitulla ${ }^{1}$. Peter A. Fasching ${ }^{1} \cdot$ Matthias W. Beckmann ${ }^{1} \cdot$ Anna-Katharin Theuser ${ }^{1}$

Published online: 17 May 2018

(c) Springer-Verlag GmbH Germany, part of Springer Nature 2018

Correction to: Archives of Gynecology and Obstetrics https://doi.org/10.1007/s00404-018-4779-4

The original version of this article contained an error in Fig. 1. It was provided in German. Please see the correct Fig. 1 translated into English here.

The original article can be found online at https://doi.org/10.1007/ s00404-018-4779-4.

Carolin C. Hack

carolin.hack@uk-erlangen.de

1 Department of Gynecology and Obstetrics, Comprehensive Cancer Center Erlangen-European Metropolitan Area Nuremberg (CCC ER-EMN), Erlangen University Hospital, Friedrich Alexander University of Erlangen-Nuremberg, Universitätsstrasse 21-23, 91054 Erlangen, Germany 


\section{Standard Operation Procedure (SOP) Integrative Medicine Consultancy Service}

Integrative medicine consultancy service

Integrative medicine consultancy service

Interdisciplinary integrative medicine conference

Integrative medicine consultancy service

Integrative medicine consultancy service
Initial appointment attended by the patient

- Questionnaire on medical history

(standard hospital questionnaire)

- Integrative medicine questionnaire (IMed questionnaire)

to collect data on complaints, current therapies, co-

medication and patient's goals

- Physical examination

- Lab results

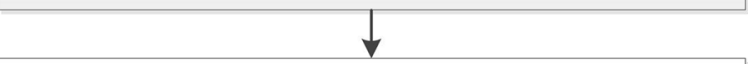

\section{After the initial appointment}

- Evaluation of the IMed questionnaire and of the standard medical history questionnaire

- Compilation of findings, medication(s) and goals

- Drug therapy safety

- Preparations for interdisciplinary integrative medicine conference

Interdisciplinary integrative medicine conference in complex cases (1x/month)

- Gynecology (general gynecology or gynecologic specialties) and/ or gynecologic oncology

- $\quad$ Naturopathic medicine

- Internal medicine

- Nutritional medicine

- Physiotherapy

- Psycho-oncology

- Acupuncture

- $\quad$ Other specialties if applicable

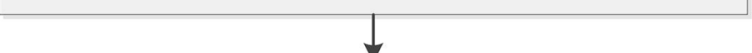

\section{Individual therapy recommendations}

Comprehensive, detailed, individual and holistic integrative medicine treatment plan

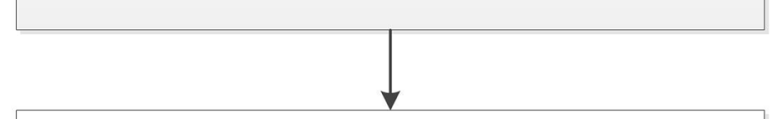

\section{Second appointment attended by the patient}

- $\quad$ Presentation of the interdisciplinary treatment recommendations

- Letter providing a summary of recommendations handed to the patient

- Additional documents (prescriptions, application instructions, nutritional recommendations, etc.)

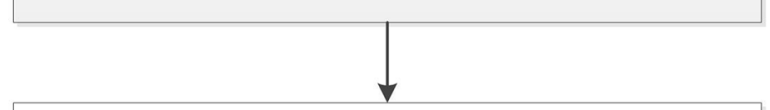

\section{Follow-up appointment (after 3-6 months)}

- $\quad$ Evaluation using the follow-up questionnaire and review of compliance

- $\quad$ Re-evaluation of the situation and modification of CAMspecific therapy if required
Flyer/ Business card

Standard questionnaire on medical history

Validated

IMed questionnaire (published in Hack et al. 2015)

Databases/ Drug therapy safety
Standardized form for interdisciplinary integrative medicine conference
Treatment recommendations / Treatment plan

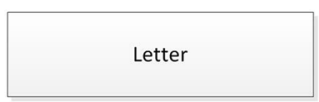

Documents

Follow-up questionnaire

Fig. 1 Modified standard operating procedure for conducting the standardized integrative medicine consultancy service 